\title{
Differential Diagnosis and Association of Acquired Immunodeficiency Syndrome and Systemic Erythematous Lupus: A Brief Review
}

\author{
Ana Paula Monteiro Gomides ${ }^{1,2 *}$, Simone Appenzeller ${ }^{3}$, Gecilmara Pileggi4, \\ Licia Maria Henrique da Mota ${ }^{1}$ \\ ${ }^{1}$ University of Brasilia (UnB), Brasília, Brazil \\ ${ }^{2}$ University Center of Brasília-UniCeub, Brasília, Brazil \\ ${ }^{3}$ Faculty of Medical Science, State University of Campinas (UNICAMP), São Paulo, Brazil \\ ${ }^{4}$ Medical School of Barretos-FACISB, São Paulo, Brazil \\ Email: *anapmgomides@gmail.com
}

How to cite this paper: Gomides, A.P.M., Appenzeller, S., Pileggi, G. and da Mota, L.M.H. (2019) Differential Diagnosis and Association of Acquired Immunodeficiency Syndrome and Systemic Erythematous Lupus: A Brief Review. Advances in Infectious Diseases, 9, 1-7.

https://doi.org/10.4236/aid.2019.91001

Received: January 11, 2019

Accepted: February 11, 2019

Published: February 14, 2019

Copyright (๑) 2019 by author(s) and Scientific Research Publishing Inc. This work is licensed under the Creative Commons Attribution International License (CC BY 4.0).

http://creativecommons.org/licenses/by/4.0/

\begin{abstract}
Acquired immunodeficiency syndrome and lupus erythematosus are multisystem diseases that can affect several organs and systems at different stages of disease evolution. Both diseases share common clinical manifestations, which may lead to diagnostic difficulties, especially at the onset of the disease. Another additional challenge is when there is an association of the two pathologies. The objective of this brief review is to describe the clinical manifestations of the diseases and to make considerations regarding the moment of onset of symptoms. Knowledge of these common manifestations and their peculiarities may alert clinicians to possible diagnoses and avoid errors in the evaluation and conduction of these patients.
\end{abstract}

\section{Keywords}

Acquired Immunodeficiency Syndrome, Systemic Lupus Erythematosus, Overlapping, Systemic Diseases

\section{Introduction}

Acquired immunodeficiency syndrome (AIDS) is a chronic, sexually transmitted infection, caused by the human immunodeficiency virus (HIV).

Systemic lupus erythematosus (SLE) is a systemic autoimmune disease that can manifest with changes in various organs and systems.

HIV infection/acquired immunodeficiency syndrome and SLE share several clinical manifestations in common. The association of these two diseases in the 
same patient is considered a real diagnostic challenge since the overlapping of symptoms can generate great difficulties in the elucidation of the medical picture and approach. HIV can mimic SLE symptoms and vice versa, which requires attention to distinguish the clinical manifestations of SLE. A high level of suspicion is required so that there is no underdiagnosis of any of these diseases [1] [2]. In 2004, Sommer et al. described a case that was miscarried as SLE for 4 years when HIV was finally diagnosed [3].

SLE and HIV are known to be systemic diseases, with involvement of several organs and consequent similarity of symptoms, which may complicate the differential diagnosis even more.The classification criteria for SLE that can help in the diagnosis (Table 1 ) in this case are often not useful sincepatients with HIV infection alone may meet 4 or more criteria necessary for the diagnosis of SLE. For this reason, some authors have already suggested a re-evaluation of the classification criteria in this population [1] [4]. Another confounding factor is the manifestation of opportunistic infections, such as candidiasis, which may occur as a complication of immunosuppression in both diseases.

The objective of this brief review is to describe the common symptomatology as well as some clinical differences between these diseases to alert the attending physician about the possible diagnoses and guide the propaedeutics.

\section{Clinical Manifestations: Similarities and Differences in Systemic Involvement}

\subsection{General Manifestations}

General symptoms such as fever, weight loss, anorexia, adynamia, lymphadenopathy and myalgia, can occur in HIV and SLE, in several phases of these diseases [1] [4].

Table 1. 1997 Update of the 1982 American College of Rheumatology Revised Criteria for Classification of Systemic Lupus Erythematosus Criterion Definition:

\begin{tabular}{l}
\hline Criterion \\
\hline Malar Rash \\
Discoid Rash \\
Photosensitivity \\
Oral ulcers \\
Arthritis \\
Serositis \\
Renal disorder \\
Neurological disorder \\
Hematologic disorder \\
Immunologic disorder \\
Antinuclear antibody \\
Onti-DNA antibody OR anti-Sm OR Antiphospholipids antibody \\
\end{tabular}




\subsection{Articular Manifestations}

Arthralgia and arthritis with varied patterns (mono, oligo or polyarticular) may occur in both SLE and HIV [1] [2] [3] [4] [5].

\subsection{Hematological Manifestations}

Anemia, leukopenia, lymphopenia and hypergammaglobulinemia are frequent manifestations in both diseases [1] [4].

\subsection{Serosites}

All types of serositis (pleuritis, pericarditis and peritonitis) have been described in HIV and are relatively common manifestations in SLE [1] [2] [3] [4] [5].

\subsection{Neurological Manifestations}

Psychoses, peripheral neuropathies, focal deficits, and stroke are neurological abnormalities shared by both diseases [6].

\subsection{Cutaneous-Mucosal Manifestations}

Oral ulcer and malar rash are classic manifestations of SLE, but may occur in HIV. Photosensitivity, characteristically described in SLE, is a common symptom in HIV and is generally considered a late manifestation in the course of infection.

The occurrence of discoid lupus erythematosus in HIV patients seems to be quite rare but has already been described in the literature [2] [7] [8].

\subsection{Renal Manifestations}

SLE and HIV present similarities to renal manifestations: both may present hematuria, proteinuria, frank nephropathy and even renal failure [6].

HIV-associated nephropathy (HIVAN) occurs most commonly in black patients and is characterized by nephrotic proteinuria, which progresses rapidly to renal failure in most cases [9]. Histologically, HIVAN is caused by focal and segmental glomerulosclerosis. Less frequently, membranoproliferative glomerulonephritis, IgA nephropathy, and thrombotic microangiopathy have been reported in patients with HIV-associated nephropathy [6]. Lupus nephritis appears to present a more varied clinical picture when compared to HIVAN and histologically there is deposition of immunocomplexes, causing the various patterns of lupus nephritis [10] [11] [12].

In 1999, Chang et al. Described 4 cases of patients with concomitant SLE and HIV who had renal changes. They were submitted to biopsy showing changes compatible with lupus nephritis in all of them. However, in 2 subjects there were still histological changes suggestive of HIVAN (tubular microcysts, podocyte hypertrophy and glomerular basement membrane collapse) and the authors concluded that there was an overlap of lupus nephritis and HIV-associated nephritis. The same authors reviewed another series of previously published 
cases in which 7 patients with HIV and SLE had renal manifestations that were diagnosed as follows after histological study: 1 patient with class III lupus nephritis and 1 patient with class IV nephritis, 2 patients with nephritis class $V$, 1 patient with $\mathrm{HIV}$-associated nephritis and 2 patients with mixed findings of lupus nephritis and HIVAN. In these two series there was a high proportion (55\%) of pediatric cases (vertical transmission of HIV), a fact that raises doubts about the mechanisms involved in the genesis of nephritis in this population [6]. This trend of onset of nephritis in the pediatric population with SLE and perinatal HIV transmission has also been described in other publications [1] [11].

Another form of nephropathy that may cause great difficulties in the differential diagnosis with SLE: lupus-like nephritis has been described in HIV infection. It is a form of glomerulopathy with presence of immunocomplexes deposits and complement in immunofluorescence, in the absence of other clinical or laboratory manifestations of lupus [13] [14]. This form of nephritis has been described in the early stages of HIV infection, soon after seroconversion, and has been reported even in transplanted kidney of HIV patients [15] [16].

8) Other manifestations

SICCA syndrome, ocular and pulmonary manifestations, thromboembolic phenomena, can manifest in a similar way in both SLE and HIV [1] [2] [3] [4] [5] [17].

Table 2 shows a summary of the clinical manifestations of the two diseases.

\section{Clinical Considerations Related to the Time of Onset of Diseases}

\subsection{Patients with HIV Infection and Subsequent Onset of SLE}

Treatment with immunosuppressants required in cases of SLE may favor the onset of manifestations of a previously undiagnosed HIV infection or worsen the course of the disease in those known to be HIV positive. This fact probably results from increased viral replication [4] [7]. Alonso and Lozada described a case of a SLE patient who developed positive HIV serology but with an undetectable viral load. After pulse therapy with cyclophosphamide due to recurrent SLE crises, there was a marked increase in viral load which was reversed with suspension of the drug [18].

Another aspect to consider is treatment related to HIV infection. Since the beginning of highly active antiretroviral therapy (HAAV), there has been great progress in the treatment of HIV-infected patients with improved morbidity and mortality. However, after recovery of immunity from treatment, new symptoms known as "immune restoration inflammatory syndrome" (IRIS) have been observed, including symptoms and new diagnoses of autoimmune diseases such as SLE . Calabrese al al described, in a literature review, 4 new cases of SLE after HAAV [19]. 
Table 2. Sistemic manifestations in AIDS and SLE [1]-[17].

\begin{tabular}{ccc}
\hline MANIFESTATIONS & AIDS & SLE \\
\hline General symptoms & Frequent & Frequent \\
Articular manifestations & Can occur & Frequent \\
Hematological manifestations & Frequent & Frequent \\
Serosites & Can occur & Frequent \\
Neurological manifestations & Can occur & Can occur \\
Cutaneous-mucosal manifestations & Frequent & Frequent \\
Renal Manifestations & Can occur (HIV-associated & Can occur (lupus \\
& nephropathy) & nephritis) \\
\hline
\end{tabular}

An additional approach already published in the literature is that the emergence of SLE in advanced stages of HIV seems to favor a milder course of lupus evolution, probably due to the role of $\mathrm{CD} 4+\mathrm{T}$ lymphocytes in its pathogenesis [5].

\subsection{Patients with SLE Who Subsequently Develop HIV Infection}

HIV infection in SLE patients appears to have a favorable impact on the progression of autoimmune disease. Several cases in the literature have described improvement and even remission of autoimmune disease after the onset of HIV. On the other hand, after initiation of HAAV therapy and improvement of the immune status, reactivation of latent symptoms or signs of SLE activity may occur [1] [4].

\subsection{Patients with Concomitant SLE and HIV}

Because it is an association that is still rare and may present similar manifestations, concomitant signs of SLE and HIV generate great difficulties in management. Several case reports show different views of how HIV can interfere with the course and manifestations of SLE and vice versa [2]. In general, the symptoms of the two conditions may coexist at the time of diagnosis or manifest in the course of evolution, and it is sometimes a real dilemma to distinguish the cause.

\section{Conclusion}

HIV infection/acquired immunodeficiency syndrome and systemic lupus erythematosus (SLE) are multisystem diseases whose diagnosis and treatment remain challenging in medical practice. The knowledge of the clinical manifestations of these diseases can alert the physician to the correct differential diagnosis and consequently the appropriate management in possible associations of both diseases.

\section{Conflicts of Interest}

The authors declare no conflicts of interest regarding the publication of this pa- 
per.

\section{References}

[1] Carugati, M., Franzetti, M., Torre, A., Giorgi, R., Genderini, A., Strambio de Castilla, F., Gervasoni, C. and Riva, A. (2013) Systemic Lupus Erythematosus and HIV Infection: A Whimsical Relationship. Reports of Two Cases and Review of the Literature. Clinical Rheumatology, 32, 1399-1405. https://doi.org/10.1007/s10067-013-2271-x

[2] Kaliyadan, F. (2008) HIV and Lupus Erythematosus: A Diagnostic Dilemma. Indian Journal of Dermatology, 53, 80.

[3] Sommer, S., Piyadigamage, A. and Goodfield, M.J. (2004) Systemic Lupus Erythematosus or Infection with HIV, or Both? Clinical and Experimental Dermatology, 29, 393-395. https://doi.org/10.1111/j.1365-2230.2004.01552.x

[4] Mody, G.M., Patel, N., Budhoo, A. and Dubula, T. (2014) Concomitant Systemic Lupus Erythematosus and HIV: Case Series and Literature Review. Seminars in Arthritis and Rheumatism, 44, 186-194.

https://doi.org/10.1016/j.semarthrit.2014.05.009

[5] Iordache, L., Launay, O., Bouchaud, O., Jeantils, V., Goujard, C., Boue, F., Cacoub, P., Hanslik, T., Mahr, A., Lambotte, O. and Fain, O. (2014) Autoimmune Diseases in HIV-Infected Patients: 52 Cases and Literature Review. Autoimmunity Reviews, 13, 850-857. https://doi.org/10.1016/j.autrev.2014.04.005

[6] Chang, B.G., Markowitz, G.S., Seshan, S.V., Seigle, R.L. and D’Agati, V.D. (1999) Renal Manifestations of Concurrent Systemic Lupus Erythematosus and HIV Infection. American Journal of Kidney Diseases, 33, 441-449. https://doi.org/10.1016/S0272-6386(99)70180-0

[7] Bhagwat, P.V., Kudligi, C., Shashikumar, B.M., Thirunavukkarasu, A. and Shendre, M.E. (2012) Extensive Discoid Lupus Erythematosus in a HIV Patient Responding to Hydroxychloroquine Monotherapy. Indian Journal of Dermatology, 57, 326-327. https://doi.org/10.4103/0019-5154.97687

[8] Vin-Christian, K., Epstein, J.H., Maurer, T.A., McCalmont, T.H. and Berger, T.G. (2000) Photosensitivity in HIV-Infected Individuals. The Journal of Dermatology, 27, 361-369. https://doi.org/10.1111/j.1346-8138.2000.tb02185.x

[9] D'Agati, V. and Appel, G.B. (1997) HIV Infection and the Kidney. Journals of the American Society of Nephrology, 8, 138-152.

[10] D’Agati, V. (1998) Renal Disease in Systemic Lupus Erythe-Matosus, Mixed Connective Tissue Disease, Sjogren's Syndrome, and Rheumatoid Arthritis. In: Jennette, J.C., Olson, J.L., Schwartz, M.M., Silva, F.G., Eds., Heptinstall s Pathology of the Kidney, Philadelphia, 541-624.

[11] Gindea, S., Schwartzman, J., Herlitz, L.C., Rosenberg, M., Abadi, J. and Putterman, C. (2010) Proliferative Glomerulonephritis in Lupus Patients with Human Immunodeficiency Virus Infection: A Difficult Clinical Challenge. Seminars in Arthritis and Rheumatism, 40, 201-209. https://doi.org/10.1016/j.semarthrit.2009.12.001

[12] Palacios, R., Santos, J., Valdivielso, P. and Márquez, M. (2002) Human Immunodeficiency Virus Infection and Systemic Lupus Erythematosus. An Unusual Case and a Review of the Literature. Lupus, 11, 60-63. https://doi.org/10.1191/0961203302lu141cr

[13] Haas, M., Kaul, S. and Eustace, J.A. (2005) HIV-Associated Immune Complex Glomerulonephritis with "Lupus-Like" Features: A Clinicopathologic Study of 14 Cases. Kidney International, 67, 1381-1390. 
https://doi.org/10.1111/j.1523-1755.2005.00215.x

[14] Tabechian, D., Pattanaik, D., Suresh, U., et al. (2003) Lupus-Like Nephritis in an HIV-Positive Patient: Report of a Case and Review of the Literature. Clinical Nephrology, 60, 187-194. https://doi.org/10.5414/CNP60187

[15] Matignon, M., Lidove, O., Dupuis, E., Walker, F., Abgrall, S. and Papo, T. (2005) A Lupus-Like Glomerulonephritis Following Acute HIV-1 Seroconversion in an African Woman. Nephrology Dialysis Transplantation, 20, 438-440. https://doi.org/10.1093/ndt/gfh631

[16] Chandran, S., Jen, K.Y. and Laszik, Z.G. (2013) Recurrent HIV-Associated Immune Complex Glomerulonephritis with Lupus-Like Features after Kidney Transplantation. American Journal of Kidney Diseases, 62, 335-338.

https://doi.org/10.1053/j.ajkd.2013.01.010

[17] Asherson, R.A., Gómez-Puerta, J.A. and Marinopoulos, G. (2005) Recurrent Pulmonary Thromboembolism in a Patient with Systemic Lupus Erythematosus and HIV-1 Infection Associated with the Presence of Antibodies to Prothrombin: A Case Report. Clinical Infectious Diseases, 41, 89-92. https://doi.org/10.1086/497369

[18] Alonso, C.M. and Lozada, C.J. (2000) Effects of IV Cyclophosphamide on HIV Viral Replication in a Patient with Systemic Lupus Erythematosus. Clinical and Experimental Rheumatology, 18, 510-512.

[19] Calabrese, L.H., Kirchner, E. and Shrestha, R. (2005) Rheumatic Complications of Human Immunodeficiency Virus Infection in the Era of Highly Active Antiretroviral Therapy: Emergence of a New Syndrome of Immune Reconstitution and Changing Patterns of Disease. Seminars in Arthritis and Rheumatism, 35, 166-174. https://doi.org/10.1016/j.semarthrit.2005.03.007

\section{List of Abbreviations}

HIV, human immunodeficiency virus

AIDS, acquired immunodeficiency syndrome

SLE, systemic lupus erythematosus

HIVAN, HIV-associated nephropathy

HAAV, highly active antiretroviral therapy

IRIS, immune restoration inflammatory syndrome 